\title{
Transatlantica
}

Revue d'études américaines. American Studies Journal

\section{Keith F. Davis, Jane L. Aspinwall, The Origins of American Photography, 1839-1885. From Daguerreotype to Dry-Plate}

(Kansas City, Mo. : Hall Family Foundation/Nelson-Atkins Museum of Art, distr. Yale Univ. Press, 2008). 358 p., 606 ill., cat., index.

\section{François Brunet}

\section{(2) OpenEdition}

\section{Journals}

Édition électronique

URL : https://journals.openedition.org/transatlantica/4346

DOI : $10.4000 /$ transatlantica.4346

ISSN : 1765-2766

Éditeur

Association française d'Etudes Américaines (AFEA)

Référence électronique

François Brunet, «Keith F. Davis, Jane L. Aspinwall, The Origins of American Photography, 1839-1885.

From Daguerreotype to Dry-Plate», Transatlantica [En ligne], 1 | 2009, mis en ligne le 02 juillet 2009,

consulté le 19 septembre 2021. URL : http://journals.openedition.org/transatlantica/4346 ; DOI :

https://doi.org/10.4000/transatlantica.4346

Ce document a été généré automatiquement le 19 septembre 2021.

\section{(†) $\odot$

Transatlantica - Revue d'études américaines est mise à disposition selon les termes de la licence Creative Commons Attribution - Pas d'Utilisation Commerciale - Pas de Modification 4.0 International. 


\section{Keith F. Davis, Jane L. Aspinwall, The Origins of American Photography, 1839-1885. From Daguerreotype to Dry- Plate}

(Kansas City, Mo. : Hall Family Foundation/Nelson-Atkins Museum of Art, distr. Yale Univ. Press, 2008). 358 p., 606 ill., cat., index.

François Brunet

1 Publié à l'occasion de l'exposition 'Developing Greatness: The Origins of American Photography $1839-1885^{\prime}$ qui a suivi la donation de la collection Hallmark au musée Neslon-Atkins, cet imposant et somptueux volume est bien plus qu'un catalogue. Son principal auteur, Keith Davis (pas de lien apparent avec le défenseur des Dallas Cowboys) a été pendant près de trente ans l'artisan de la collection Hallmark avant de l'accompagner à Kansas City, et il ne fait pas mystère de son ambition : «to provide a new template for the appreciation of early American photography as a whole » (10). Il s'inscrit ainsi dans la lignée des Robert Taft, Beaumont Newhall, Floyd et Marion Rinhart, Richard Rudisill, William Welling, Martha Sandweiss, John Wood, dont les ouvrages avaient en leur temps déjà labouré un continent "early photography" apparemment inépuisable aux Etats-Unis. Bien qu'une précédente exposition de la Hallmark Collection ait traité du vingtième siècle (voir l'ouvrage associé, non moins imposant: An American Century of Photography: From Dry-Plate to Digital, The Hallmark Photographic Collection, 1994) et que Keith Davis revendique non sans raison la valeur cumulée des deux livres comme panorama de l'histoire photographique américaine, cette nouvelle publication confirme la singularité de ce dix-neuvième siècle américain, qui explique pour partie ce mystère historiographique qui veut que personne n'ait jusqu'ici tenté une véritable synthèse de l'histoire de la photographie aux Etats-Unis.

2 Le continent photographique du dix-neuvième, ici décrit dans un luxe inouï de détails, n'a, de fait, pas grand-chose à voir avec la physionomie habituellement dépeinte de la photographie du vingtième siècle, où dominent des catégories stylistiques ou 
génériques (photographie "pure", "documentaire", «de rue », etc.) associées à des grands noms. Suivant la tradition propre aux dix-neuviémistes américanistes, Keith Davis et Jane Aspinwall adoptent un découpage où la chronologie technologique (daguerréotype / filière verre-papier / transition de la plaque sèche au film) épouse étroitement l'histoire nationale et sa transcription en termes thématique. Après trois chapitres particulièrement nourris sur l'ère du daguerréotype, vient un chapitre ("The Face of a Nation ») sur l'importance sociale et culturelle du portrait dans une période de quête identitaire et démocratique. Puis, suivant un chapitre intermédiaire sur l'essor de la photographie sur papier, viennent trois chapitres (sur la guerre civile, la photographie de paysage et de promotion, enfin l'expansion de la culture visuelle de masse à la fin du siècle) dont la succession suggère l'isomorphisme qui lie aux EtatsUnis histoire nationale et histoire des images. L'art de la photographie, au sens habituel de démiurgie perceptive, n'a guère de place dans ce récit, et du même coup cette histoire très américaine ne communique qu'à grand-peine avec l'histoire internationale de la photographie ancienne, où tendent jusqu'ici à dominer petits et grands maîtres inspirés par les genres picturaux.

3 Même admirablement articulés et impeccablement mis à jour sur la base de l'historiographie récente, ces traits constituent un paysage bien connu et ils ne font donc pas l'originalité du catalogue. Ce qui est remarquable ici est plutôt une série d'études de détail insérées dans ce canevas, et qui, appuyée sur une iconographie abondante et superbement reproduite, enrichit notablement notre connaissance du monde ancien des images modernes. Cet enrichissement concerne surtout «l'ère » du daguerréotype, univers foisonnant (voir par exemple le site de la Daguerreian Society) qui demeure dans cet ouvrage la grande spécificité américaine. Dans le premier chapitre, qui traite des pionniers et de leur tendance précoce à la professionnalisation, deux " études de cas " sont consacrées respectivement aux studios de Southworth et Hawes (les Bostoniens raffinés, portraitistes de la bonne société et amateurs d'expériences formellles, et déjà bien connus, surtout depuis la grande exposition "Young America » de Rochester en 2005 et le monumental catalogue qui en est issu) et à l'éphémère et grandiose chaîne d'établissements daguerriens de l'émigré gallois John Plumbe. Il y a là de bons exemples des progrès d'une muséographie spécialisée déjà assez sûre de ses bases pour s'aventurer avec succès dans l'exploration d'individualités plutôt obscures aux yeux du grand public. Le chapitre III, sur le métier de daguerréotypiste, résume à lui seul la distance qui sépare, au XIXe siècle, la photographie américaine, fière de ses recettes et de son organisation technicocommerciale, de photographies européennes structurellement en marge des institutions culturelles et artistiques - et aussi bien celle qui sépare une historiographie américaine ou américaniste qui met l'organisation matérielle et professionnelle au premier rang des facteurs historiquement structurants, d'une historiographie française en particulier, encore souvent engluée dans une vision extraordinairement idéaliste et styliste des arts de l'image (voir notamment la section "The Language of Light: Commerce and Art »). Le chapitre IV, "The Face of a Nation ", est en lui-même une monographie de très belle facture sur l'homothétie qui relie les mille et une applications et variations du daguerréotype (dominées à 95\% par le commerce du portrait) et la réalité diverse et mouvante de la société américaine du milieu du siècle. Seul un pays dont la mémoire nationale se définit, au moins populairement, dans un cadre chronologique voisin de celui de l'histoire de la photographie (si Jefferson, Washington ou La Fayette n'ont pas connu le daguerréotype, 
pensons qu'Andrew Jackson, John Quincy Adams, Henry Clay, Daniel Webster, sans parler d'Emerson, Lincoln, Frederick Douglass, etc. ont été abondamment photographiés) peut être en mesure d'accorder tant d'importance et d'efforts critiques à la reconstitution d'une imagerie contemporaine de la nation. Celle-ci a ses grandeurs (le portrait de profession ou occupational portrait en est une évidente, ici remarquablement analysée et illustrée) et ses lacunes plus ou moins criantes (Indiens et Noirs, ces derniers esclaves ou non, sont globalement peu et mal photographiés, mais les recherches redoublées des deux dernières décennies dans ces domaines ont néanmoins mis au jour beaucoup d'images encore insoupçonnées en 1980). Une fois de plus, mais de façon magistrale, est ici dessinée la voie d'une histoire des images qui s'entend d'abord comme histoire de pratiques sociales et dont l'attrait esthétique très vif - se comprend pleinement dans ce cadre social, sans avoir à être codé dans un vocabulaire idéaliste ou romantique.

INDEX

Thèmes : Recensions

\section{AUTEUR}

FRANÇOIS BRUNET

Université Paris Diderot - Paris 7 